\title{
la composición de los cementos puzolánicos y su resistencia a las aguas sulfatadas
}

\author{
Prof. A. RIO y Dres. A. CELANI y L. ANGELETTI
}

(«L'Industria Italiana del Cemẹnto», XXXI, núm. 4, abril 1961, pág. 184.)

El creciente desarrollo en el empleo de los cementos puzolánicos en el mundo, debido a los buenos resultados obtenidos en la aplicación de estos aglomerantes, no ha ido acompañado del correspondiente progreso de nuestros conocimientos sobre el mecanismo de la acción puzolánica, sobre todo en lo que se reflere a la notable resistencia química de estos conglomerantes.

El estudio de las transformaciones, mediante las cuales el cemento Portland, asociado con la puzolana, se hace resistente al ataque de las más comunes aguas agresivas naturales, constituye, continuamente, un problema de notable interés, no sólo práctico, sino también científico, por sus reflejos en el campo de la química de los aglomerantes hidráulicos.

Las viejas teorias formuladas en el pasado sobre el tema deben conservarse, al menos en parte, aunque se hayan superado en los últimos años a la luz de los resultados obtenidos en el estudio de los productos de hidratación de las pastas de cemento.

El conocimiento actual de la química de los conglomerantes hidráulicos no permite relacionar las diferencias encontradas o, con mucha probabilidad, supuestas entre las fases que constituyen la pasta hidratada de los cementos Portland y puzolánicos con su diversa resistencia química. Desde un punto de vista cualitativo, resulta (1) que la constitución química de las pastas de los dos tipos de cemento es, aproximadamente, la misma, con excepción del hidróxido cálcico, presente en los cementos Portland y prácticamente ausente en los puzolánicos.

En ambas pastas están presentes silicatos hidratados de calcio de tipo tobermorítico, etringita, aluminato tetracálcico y eventuales cristales mixtos de este último compuesto con el correspondiente ferrito y con la forma monosulfática.

Otra diferencia probable podría tener relación con la composición de las fases tobermoríticas, en el sentido de que mientras es presumible que en el cemento Portland se encuentre una tobermorita con el máximo contenido de óxido de calcio estable en una solución de cal próxima a la saturación, en las pastas de cemento puzolánico, donde en un tiempo relativamente corto casi todo el hídróxido cálcico de la hidrólisis lo ha fljado la puzolana, la tobermorita deberá ser de bajo contenido en óxido de calcio, en equilibrio con una solución mucho más pobre en cal que la anterior.

Fstos hechos, confirmados recientemente también por Lea en el IV Congreso Internacional de Química del Cemento de Wáshington (2), no justifican el distinto comportamiento en obra de los dos conglomerantes y hacen presumir que la mayor resistencia química de los cementos puzolánicos depende, no tanto de las diversas fases presentes en las pastas hidratadas como de su relación cuantitativa que dé a la pasta endurecida una estructura particular más resistente. 
Tales deducciones han encontrado numerosas conflrmaciones en los resultados obtenidos por diversos investtgadores en condiciones experimentales muy diferentes entre sí $(3,4$ y 5). Estos resultados, en su conjunto, hacen pensar que la causa principal de la resistencia química de las pastas puzolánicas debe atribuirse a la mayor impermeabilidad $\mathrm{y}$, en particular, a la notable acción protectora que sobre las fases presentes ejercen los geles de nueva formación, obstaculizando la difusión de los iones y los cambios con la solución de contacto.

Resultaria, por Io tanto, que la acción puzolánica se maniftesta a través de una modificación de las caracteristicas físicas de la pasta conglomerante provocada por los compuestos de nueva formación originados a consecuencia de la reacción química de la cal de hidrólisis con los constituyentes activos de la puzolana. Es evidente que la resistencia quimica de un cemento puzo-

lánico dependerá de la cantidad de estos geles que pueden justificar una acción protectora. Tal acción, útil en toda forma de agresión, es indispensable en el caso del ataque de aguas sulfatadas, con las que pueden reacclonar, además de los aluminatos procedentes del clínker, los que pueden formarse de la misma puzolana.

A este respecto, ya se ha puesto en evidencia (6) la influencla determinante del contenido en sillce reactiva de la adición puzolánica y cómo la resistencla química de los cementos puzolánicos se encuentra en estricta conexión con la relación que puede establecerse entre la silice y la alúmina reactivas presentes en dichos cementos. Resultaria, por tanto, posible graduar, mediante tales valores, la resistencia química a las aguas sulfatadas de un cemento puzolänico, hasta hacerlo resistente a las severas condiciones del ensayo Anstett, el cual, aunque lejos de conservar las condiciones normales de ataque, es todavía significativo al poner en evidencia una absoluta resistencia especifica del proplo aglomerante.

En los ultimos tiempos este argumento se ha confirmado, en el Laboratorio de los autores, mediante una serie de pruebas sistemáticas de las que se exponen los resultados por la contribución que puedan aportar a una rápida valoración de la resistencia quimica de los cementos puzolánícos.

A partir de dos clínkeres de cemento Portland, uno normal y otro del tipo férrico, y de dos materiales puzolánicos típicos, cuyas características se reflejan en la tabla 1, se han preparado una serie de 20 cementos diferenciales por una relactón $\mathrm{S} 1 \mathrm{~A} / \mathrm{Al}_{2} \mathrm{O}$, reactiva creciente. Las mezclas se han obtenido premoliendo los diversos componentes a Ia misma finura, correspondiente a un residuo de 17.000 mallas $/ \mathrm{cm}^{2}$ de cerca del $15 \%$.

TA B L A N U M . 1

Composioion quimica de los materiales empleados

A) Clinker.

\begin{tabular}{|c|c|c|c|c|c|c|c|c|c|c|c|c|c|c|c|}
\hline & p. p.e. & $\underset{(\%)}{\mathbf{S} 1 \mathbf{O}_{2}}$ & $\begin{array}{l}\mathrm{Fe}_{e_{2} \mathrm{O}_{2}} \\
(\%)\end{array}$ & $\underset{(\%)}{\mathrm{AbO})_{2}}$ & $\underset{(\%)}{\mathrm{CaO}}$ & $\underset{(\%)}{M}$ & $\begin{array}{l}\mathbf{5 0} ; \\
(\%)\end{array}$ & M. I. & M. s. & M. F. & $\underset{(\%)}{\mathbf{C}_{3} \mathbf{S}}$ & $\begin{array}{l}\mathrm{C}, \mathrm{S} \\
(\%)\end{array}$ & $\begin{array}{l}C_{A A} \\
(\%)\end{array}$ & $\underset{(\%)}{\text { CuAF }}$ & $\begin{array}{c}\text { Grado } \\
\text { de } \\
\text { saturaolon } \\
(\%)\end{array}$ \\
\hline linker normal ....... & 1,60 & $21,-$ & 2,76 & 6,34 & 66,50 & $1,-$ & 0,80 & 2,21 & 2,30 & 2,29 & 51,58 & 21,32 & 12,13 & 8,40 & 97,60 \\
\hline fundentes & 0,07 & 22,32 & $4,-$ & 4,34 & 65,75 & 1,34 & 0,87 & 2,14 & 2,67 & 1,08 & 59,52 & 19,41 & 4,74 & 12,17 & 93,64 \\
\hline
\end{tabular}

B) Matertales puzolánicos.

\begin{tabular}{|c|c|c|c|c|c|c|c|c|c|}
\hline & p. p.o. & $\underset{(\%)}{\mathrm{SiO}_{2}}$ & $\underset{(\%)}{\mathrm{Fe}_{2} \mathrm{O}_{3}}$ & $\begin{array}{c}\mathbf{A l}_{\mathbf{2}} \mathbf{O}_{\mathbf{M}} \\
(\%)\end{array}$ & $\begin{array}{l}\mathrm{CaO} \\
(\%)\end{array}$ & $\begin{array}{c}\text { Restduo } \\
\text { puzolinico } \\
(\%)\end{array}$ & $\underset{(\%)}{\operatorname{sio}_{2}}$ & $\begin{array}{c}\mathrm{R}_{2} \mathrm{O}_{9} \\
\text { soluble } \\
(\%)\end{array}$ & 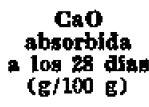 \\
\hline $\begin{array}{lllllll}\text { Puzolana } & \text { A } & \ldots & \ldots & \ldots & \ldots & \\
\text { Puzolana } & \text { B } & \ldots & \ldots & \ldots & \ldots & \ldots\end{array}$ & $\begin{array}{r}10,12 \\
5,63\end{array}$ & $\begin{array}{l}85,56 \\
44,92\end{array}$ & $\begin{array}{l}0,46 \\
9,70\end{array}$ & $\begin{array}{r}2,92 \\
17,58\end{array}$ & $\begin{array}{r}0,58 \\
11,02\end{array}$ & $\begin{array}{r}2,50 \\
13,10\end{array}$ & $\begin{array}{l}50,16 \\
18,90\end{array}$ & $\begin{array}{r}2,22 \\
15,04\end{array}$ & $\begin{array}{l}51,27 \\
21,33\end{array}$ \\
\hline
\end{tabular}

(*) Valores determingdos según Florentin, después de conservactón de veintiocho días en contacto con cal.

La clasiflcación, en cuanto a sílice, alúmina y cal reactivas, se ha establecido determinando las cantidades de los tres óxidos solubles al ataque Forentín, presente en las pastas hidratadas y conservadas en las condiciones del ensayo de puzolanicidad (7) propuesto para el control de los cementos puzolánicos en Italia y aceptado en organismos internacionales. 
Hemos reservado el omitir la acción desarrollada por el óxido de hierro, ya que no existen datos preclsos sobre su participación en la reacción con el hidróxido cálcico que, aunque tuviera lugar, deben ocurrir con velocidad muy inferior a la de la silice y la alúmina presentes.

Por otra parte, también los clínkeres probados en una sulfatación preliminar, llevada paralelamente, han demostrado que la cantidad de $\mathrm{SO}$, fjado en las diversas condiciones ha resultado siempre proporcional a la cantidad de alúmina presente.

En la tabla 2 se encuentra la clasificación de los cementos en cuanto a las cantidades de sílice, alúmina y cal solubles encontradas en las pastas hidratadas y conservadas como hemos dicho antes que, con buena aproximación, considerábamos en correspondencia con las cantidades reactivas de los tres óxidos presentes en los cementos.

Las relaciones silice-alúmina reactivas varian desde un valor minimo de 2,44 para la muestra número 1 a un valor máximo de 10,86 para la muestra número 20. Las muestras números 4 y 13 corresponden a los dos clínkeres puros y se caracterizan por una relación $\mathrm{StO}_{2} / \mathrm{Al}_{2} \mathrm{O}$, de 3,33 y 5,59 , respectivamente.

T A B L A N U M , 2

Composictones de los cementos en estudio, calcaladas según los oxidos solnbles despaés del ataque Floxentín de pastas hidratadas y conservadas según la técnica del ensayo $\mathbf{y}$ puzolanicidad.

(Valores referidos a muestras calcinadas.)

\begin{tabular}{|c|c|c|c|c|c|c|c|}
\hline \multirow{2}{*}{ MUESTAS } & \multicolumn{3}{|c|}{$\begin{array}{c}\text { OXIDOS SOLUBLES SEGUN EL } \\
\text { METODO WLOAENTLN } \\
(\%)\end{array}$} & \multicolumn{3}{|c|}{ OXIDOS, $\underset{\text { DE SU SUMA }}{\text { EXPREADOS }}$} & \multirow{2}{*}{ 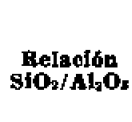 } \\
\hline & sion & $\mathbf{A 1}_{2} \mathbf{O}_{9}$ & $\mathrm{GaO}$ & $\mathrm{slO}_{2}$ & $\mathbf{A l}_{3} \mathbf{O}_{\mathbf{2}}$ & $\mathrm{GaO}$ & \\
\hline $\begin{array}{r}1 \\
2 \\
3 \\
4 \\
5 \\
6 \\
7 \\
8 \\
9 \\
10 \\
11 \\
12 \\
13 \\
14 \\
15 \\
16 \\
17 \\
18 \\
19 \\
20\end{array}$ & $\begin{array}{l}20,89 \\
22,45 \\
20,75 \\
20,76 \\
23,52 \\
22,55 \\
24,85 \\
24,57 \\
24,20 \\
25,96 \\
26,84 \\
27,41 \\
21,75 \\
28,01 \\
28,20 \\
24,86 \\
28,36 \\
29,32 \\
28,62 \\
31,50\end{array}$ & $\begin{array}{l}\mathbf{8 , 5 6} \\
\mathbf{7 , 3 0} \\
\mathbf{6 , 6 5} \\
\mathbf{6 , 2 4} \\
\mathbf{7 , 0 3} \\
\mathbf{6 ,} \mathbf{6} \\
\mathbf{6 , 1 7} \\
\mathbf{5 , 6 4} \\
\mathbf{5 , 4 1} \\
\mathbf{5 , 7 8} \\
\mathbf{4 , 9 9} \\
\mathbf{5 , 0 8} \\
\mathbf{3 , 8 9} \\
4,93 \\
4,45 \\
\mathbf{3 , 5 8} \\
\mathbf{4 , 0 1} \\
\mathbf{3 , 7 7} \\
\mathbf{3 , 2 2} \\
\mathbf{2 , 9 0}\end{array}$ & $\begin{array}{l}44,04 \\
43,80 \\
44,27 \\
64,88 \\
43,86 \\
44,05 \\
43,57 \\
58,93 \\
44,- \\
43,75 \\
43,69 \\
43,87 \\
64,19 \\
52,07 \\
43,50 \\
58,51 \\
43,89 \\
43,87 \\
53,42 \\
43,80\end{array}$ & $\begin{array}{l}28,42 \\
30,53 \\
28,94 \\
22,60 \\
31,60 \\
31,06 \\
33,32 \\
27,56 \\
32,87 \\
34,39 \\
35,54 \\
35,92 \\
24,21 \\
32,95 \\
37,03 \\
28,59 \\
37,19 \\
38,09 \\
33,37 \\
40,28\end{array}$ & $\begin{array}{r}11,64 \\
9,93 \\
9,27 \\
6,79 \\
9,44 \\
8,26 \\
\mathbf{8 , 2 7} \\
\mathbf{6 , 3 3} \\
\mathbf{7 , 3 5} \\
\mathbf{7 , 6 6} \\
\mathbf{6 , 6 1} \\
\mathbf{6 , 5 8} \\
\mathbf{4 , 3 3} \\
\mathbf{5 , 8 0} \\
\mathbf{5 , 8 4} \\
\mathbf{4 , 1 2} \\
\mathbf{5 , 2 6} \\
\mathbf{4 , 9 0} \\
\mathbf{3 , 7 7} \\
\mathbf{3 , 7 1}\end{array}$ & $\begin{array}{l}59,94 \\
59,55 \\
61,79 \\
70,61 \\
58,96 \\
60,67 \\
58,41 \\
66,11 \\
59,77 \\
57,95 \\
57,85 \\
57,50 \\
71,46 \\
\mathbf{6 1 , 2 5} \\
\mathbf{5 7 , 1 2} \\
\mathbf{6 7 , 2 9} \\
\mathbf{5 7 , 5 5} \\
\mathbf{5 7 , 0 1} \\
\mathbf{6 2 , 6 6} \\
\mathbf{5 6 , 0 1}\end{array}$ & $\begin{array}{r}2,44 \\
3,07 \\
3,12 \\
\mathbf{3 , 3 3} \\
3,34 \\
\mathbf{3 , 7 6} \\
4,03 \\
4,35 \\
4,47 \\
\mathbf{4 , 4 9} \\
\mathbf{5 , 3 8} \\
\mathbf{5 , 4 6} \\
\mathbf{5 , 5 9} \\
\mathbf{5 , 6 8} \\
\mathbf{6 , 3 4} \\
\mathbf{6 , 9 3} \\
\mathbf{7 , 0 7} \\
\mathbf{7 , 7 7} \\
\mathbf{8 , 9 0} \\
10,86\end{array}$ \\
\hline
\end{tabular}

En la tabla 3 se encuentran los datos de resistencias mecánicas, según la norma italiana de los 20 cementos considerados, resistencias que pueden considerarse buenas para todas las muestras.

Para controlar la resistencia quimica de estas muestras a las aguas sulfatadas se han adoptado dos métodos distintos empleados anterlormente en nuestro laboratorio con el mísmo fin; a saber:

El examen del comportamiento, en el tiempo, de pastas porosas preparadas con las diversas muestras y sumergidas, después del adecuado curado en agua normal, en la solución agresiva;

El ensayo de resistencia química Anstett.

El primer método nos da un índice químico del aglomerante que responde mejor a las condiclones de empleo; pero, sin embargo, requieren mucho tiempo y no permiten una discriminación precisa, como todos los métodos que se basan en una valoración subjetiva. 
T A B L A $\mathbf{N}$ U $\mathbf{M}$. 3

Resistencias meeánicas en mortero, según la norma italiana, de los cementos estudiados.

MUESTR
1
2
3
4
5
6
7
8
9
10
11
12
13
14
15
16
17
18
19
20

\begin{tabular}{|c|c|c|}
\hline \multicolumn{3}{|c|}{ T R $\underset{\left(\mathrm{kg} / \mathrm{cm}^{2}\right)}{\operatorname{ACO}} \operatorname{CN}$} \\
\hline 3 dias & 7 dias & 28 dfas \\
\hline 28 & 31 & 37 \\
\hline 27 & 32 & 39 \\
\hline 26 & 30 & 39 \\
\hline 29 & 32 & 38 \\
\hline 29 & 32 & 40 \\
\hline 26 & 32 & 39 \\
\hline 28 & 34 & 42 \\
\hline $\mathbf{3 0}$ & 37 & 43 \\
\hline 27 & 34 & 40 \\
\hline 29 & 35 & 43 \\
\hline 30 & 36 & 45 \\
\hline 28 & 34 & 42 \\
\hline 30 & 33 & 38 \\
\hline 28 & 38 & 44 \\
\hline 26 & 37 & 45 \\
\hline 29 & 37 & 44 \\
\hline 30 & 36 & 43 \\
\hline 29 & 35 & 44 \\
\hline 29 & 40 & 45 \\
\hline 27 & 36 & 45 \\
\hline
\end{tabular}

\begin{tabular}{|c|c|c|}
\hline \multicolumn{3}{|c|}{ COHPRESION } \\
\hline 3 dias & 7 dias & 28 dias \\
\hline 424 & 536 & 700 \\
\hline $\begin{array}{l}721 \\
442\end{array}$ & 568 & 726 \\
\hline 430 & 550 & 716 \\
\hline 610 & 680 & 756 \\
\hline 430 & 576 & 720 \\
\hline 450 & 580 & 744 \\
\hline 430 & 584 & 742 \\
\hline 596 & 730 & 850 \\
\hline 446 & 600 & 740 \\
\hline 440 & 630 & 768 \\
\hline 426 & 628 & 790 \\
\hline 434 & 626 & 754 \\
\hline 630 & 690 & 772 \\
\hline 520 & 692 & 796 \\
\hline 428 & 650 & 826 \\
\hline 620 & 755 & 870 \\
\hline 450 & 650 & 784 \\
\hline 444 & 648 & 810 \\
\hline 536 & 700 & 820 \\
\hline 434 & 630 & 800 \\
\hline
\end{tabular}

Los resultados que se obtengan con este método dependen, adémás, como es lógico, de la relación aglomerante/arena adoptada y de la granulometria de esta ultima, por lo que los resultados se influencian mucho por el grado de compacidad de las probetas. En otros términos, con este método se mide, de un modo complejo, la resistencia química específlca del congIomerante y la aportación que a ella puede dar la impermeabilidad del exterior, variable con el modo de realización de la probeta.

El ensayo de Anstett mide, por el contrario, sólo la resistencia intrínseca del cemento, sin tener en cuenta las defensas exteriores de la obra al ataque; por ello, aparte de por su elevada concentración agresiva, este ensayo está lejos de cumplir las condiclones prácticas que tienen lugar, generalmente, en el uso de los cementos. Sin embargo, permite, mediante la medida precisa de la expansión en el tlempo, una valoración más exacta de la resistencia química de un cemento, y si, en efecto, la resistencia al ensayo anstett de un conglomerante no es una condieión necesaria para su buen comportamiento en obra, es evidente que responder al ensayo es una segura garantía de éxito.

La confección del mortero poroso para el ensayo de conservación en solución agresiva se ha hecho con una relación cemento/arena de $1: 5$, empleando una arena cuarzosa monogranular de unos 0,2 mm para dar a las probetas una notable porosidad. Después de una conservación de veintiocho dias en agua potable, las probetas se han sumergido en el líquido agresivo, constituido por una solución al $3 \%$ de sulfato magnésico, que se ha renovado semanalmente.

Para la técnica del ensayo Anstett modificado por Blondiau, nos remitimos al trabajo original de este último (8). Sólo recordaremos que, según el ensayo, la máxima expansión tolerada, después de tres meses de conservación, es la que corresponde a un aumento en el diámetro de las probetas de $1,3 \%$.

Los resultados obtenidos se reúnen en las tablas 4 y 5 , refiriéndose los valores de la expansín encontrados en el ensayo Anstett $\mathrm{y}$ describiéndose, con el tlempo, el estado de las probetas en contacto con la soluefón agrestva.

De dichas tablas resulta evidente que Ia resistencia química de los diversos cementos es pro-

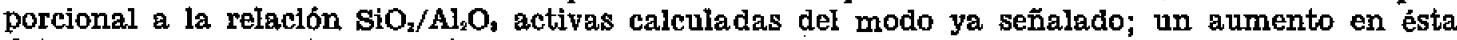
determina una resistencia quimica creciente, de tal manera que valores superiores a 5 permiten al aglomerante superar la propia prueba del ensayo de Anstett.

Relaciones inferiores, como se observa en las probetas 1 a 10 , no responden al ensayo, y la expansión producida se encuentra slempre relacionada con el valor de dicha relación. Para valores superiores a en la relación $\mathrm{SiO}_{2} / \mathrm{Al}_{2} \mathrm{O}_{2}$, la resistencia química al ensayo de Anstett es prácticamente absoluta, incluso con conservación de un año y más.

Naturalmente, la resistencia química de las diversas muestran depende también de la cantidad, en valor absoluto, de la alúmina presente; sin embargo, la relación $\mathrm{SiO}_{3} / \mathrm{Al}_{2} \mathrm{O}_{3}$ empleada permite una valoración más gradual y precisa de tal resistencia, dada la maniflesta acción protectora de la silice presente. 
T A B L A N U M , 4

Comportamiento de las probetas de mortero poroso $1: 5$ al ataque de 1a solución agresiva.

(Probetas $40 \times 40 \times 160 \mathrm{~mm}$. Soluclón de SOMg, 7Ho al $3 \%$ )

$\begin{array}{cc}\text { MUEsThAs } & 1 \text { mes } \\ 1 & \text { Intacto } \\ 2 & " \\ 3 & \text { Primera fisura } \\ 4 & \text { Intacto } \\ 5 & " \\ 6 & " \\ 7 & " \\ 8 & " \\ 9 & " \\ 10 & " \\ 11 & " \\ 12 & " \\ 13 & " \\ 14 & " \\ 15 & " \\ 16 & " \\ 17 & " \\ 18 & " \\ 19 & \end{array}$
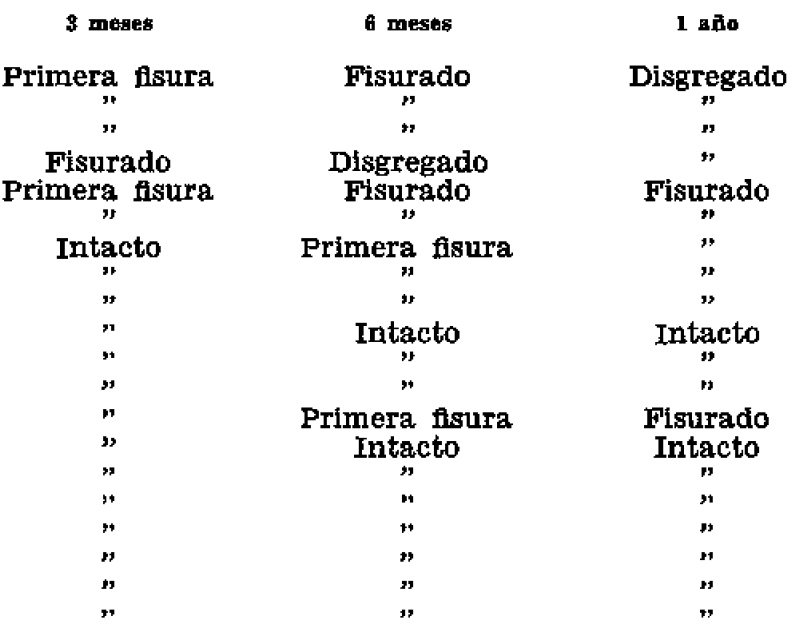

T A B L A N U M . 5

Resultado del ensayo Anstett

MUESTRAS

AUMENTO PORGENTUAL DEL DIAMETRO A DIVERSOS PERIODOS DE CONSER YACION

\begin{tabular}{|c|c|c|c|c|c|c|c|}
\hline & g dias & $7 \mathrm{dfas}$ & 14 dras & 28 días & 3 meses & 6 meses & 1 gx̃o \\
\hline 1 & 0,68 & 4,80 & 11,05 & 19,80 & $20,-$ & $21,-$ & $23,-$ \\
\hline 2 & 1,57 & 6,34 & 11,34 & 20 & 21, & 21,50 & $22,-$ \\
\hline 3 & 0,11 & 1,57 & 3,71 & 6,16 & 13,75 & 15,60 & $18, \ldots$ \\
\hline 4 & 1,00 & 3,75 & 11,75 & 13,60 & 19,25 & $21,-$ & $24,-$ \\
\hline 5 & 0,42 & 2,17 & 4,68 & 9,30 & 13,60 & 15,10 & $17,-$ \\
\hline 6 & 0,50 & 1,71 & 6,70 & 8,60 & $13,-$ & $15,-$ & 16,50 \\
\hline 7 & 0,20 & 1,33 & 2,20 & 3,30 & $4, \ldots$ & $4,-$ & $4,-$ \\
\hline 8 & 0,10 & 0,87 & $1,-$ & 1,75 & 2,80 & 3,50 & 3,50 \\
\hline 9 & 0,11 & 0,45 & $1,-$ & 1,19 & 2,60 & 3,50 & 3.50 \\
\hline 10 & 0,03 & 0,14 & 0,24 & 0,34 & 1,20 & 1,80 & $2,-$ \\
\hline 11 & 0,06 & 0,18 & 0,22 & 0,28 & 0,28 & 0,30 & 0,30 \\
\hline 12 & 0,05 & 0,15 & 0,21 & 0,24 & 0,80 & 0,80 & 0,80 \\
\hline 13 & 0,04 & 0,04 & 0,24 & 0,56 & 1,10 & 5,94 & 7,56 \\
\hline 14 & 0,03 & 0,14 & 0,20 & 0,20 & 0,20 & 0,50 & 0,70 \\
\hline 15 & 0,03 & 0,05 & 0,06 & 0,08 & 0,08 & 0,08 & 0,10 \\
\hline 16 & 0 & $0,-$ & 0,04 & 0,07 & 0,30 & 0,50 & 0,50 \\
\hline 17 & 0,05 & 0,15 & 0,21 & 0,24 & 0,27 & 0,27 & 0,30 \\
\hline 18 & 0,05 & 0,15 & 0,15 & 0,15 & 0,15 & 0,15 & 0,15 \\
\hline 19 & $0,-$ & $0,-$ & $0,-$ & 0,05 & 0,05 & 0,05 & 0,05 \\
\hline 90 & $0,-$ & $0,-$ & $0,-$ & 0,05 & 0,05 & 0,05 & 0,07 \\
\hline
\end{tabular}

E1 comportamiento de las probetas de mortero poroso, en nuestras condiciones experimentales, lleva a conclusiones muy similares a las del ensayo Anstett, comprobación interesante a la hora de juzgar los dos métodos experimentales. Además, el primer cemento de la serie cuyas probetas resultan intactas después de un af́o de conservación es el número 10, caracterizado por una relaclón $\mathrm{SiO}_{2} / \mathrm{Al}_{2} \mathrm{O}_{3}=4,50$, no muy distinto de los valores encontrados para el primer cemento de la serle resistente al ensayo Anstett.

También con las probetas en mortero poroso es posible una graduación de la resistencla quimica de los cementos en función de la relación indicada; asi, en tanto que las probetas con una relación próxima a 3 resultan lesionadas a partir de tres meses, las de relación superior a 4 aparecen lesionadas después de seis meses e incluso, después de un año de conservación en la solución agresiva. 
Haremos una mención especlal a la muestra número 13 , constituída de Portland férrico de módulo silícico elevado. Este cemento, caracterizado por una relación $\mathrm{SiO}_{2} / \mathrm{Al}_{2} \mathrm{O}_{3}$ de 5,6 , aunque en el conjunto muestra una buena resistencia química, en periodos de conservación largos presenta valores de expansión y de corrosión superiores a los de los cementos números 11 y 12 que lo preceden en la serle, con relaciones $\mathrm{SiO}_{2} / \mathrm{Al}_{2} \mathrm{O}_{3}$ menores.

La posibilidad de poder valorar la resistencia química de un cemento puzolánico por la relación que puede establecerse entre la silice y la alúmina reactivas presentes después de la oportuna maduración en el aglomerante hidratado, se ha conflrmado con unas experiencias hechas con la misma técnica sobre otra serie de cementos formada por 34 muestras numeradas de 21 a 54 con una telación $\mathrm{S}_{3} \mathrm{O}_{2} / \mathrm{Al}_{2} \mathrm{O}_{4}$ activas creciente.

Los cementos que forman esta segunda serie se han obtenido con clínkeres y puzolanas de origen diverso y expuesto al control de la resistencia según el ensayo de Anstett. La determinación de silice, alúmina y cal activas se ha llevado a cabo, como para la serie precedente, determinando la cantidad de estos oxidos solubles por el ataque Florentín de la pasta hidratada y conservada según el ensayo de puzolanictdad.

T A B L A N V M .

Segunda serie de ensayos

Composiclón de Ios cementos en estudio calculada hasada en los óxidos solubles después del ataque Florentín de la pasta hidratada y conservada según la técnlca del ensayo de pazolanicidad

(Valores referidos a muestras desecadas)

MUESTRAS

METODO FOORENTTN
$\mathrm{SiO}_{2} \quad \mathrm{Al}_{2} \mathrm{O}_{3}$

18,51

19,35

20,03

18,70

20,20

19.56

20,80

20,68

20,68

20,85

24,74

21,44

25,07

22,20

23,20

29,84

24,58

22,15

30,06

30,0 d

21,2

31,65

25,59

26,43

25,92

32,32

31,69

24,13

22,54

26,16

30,34

31,93

32,54

25,02

8,55
8,80
8,47
7,77
$\mathbf{8 , 3 9}$
7,95
7,02
6,95
6,88
5,60
6,26
4,99
5,14
4,14
4,32
5,37
4,35
3,40
4,51
4,25
2,86
3,93
3,11
3,13
2,98
3,62
3,54
2,68
2,48
2,86
3,04
2,81
2,76
1,35

8,55

8,80

7,77

8,39

7,95

6,02

6,88

5,60

6,26
4,99

5,14

4,14

4,32
5,37

4,35

$\mathbf{3 , 4 0}$

4,51

2,86

3,93

$\mathbf{3 , 1 1}$

2,98

3,62

3,64
2,68

2,48

2,86

3,04

2,76

1,35

\section{1,58}

34,24

41,14

31,45

42,45

34,09

43,94

41,93

42,23

62,90

42,42

54,52

42,23

62,80

65,70

33,11

40,40

64,80

32,97

44,56

49,45

41,23

47,25

48,10

45,60

32,22

39,10

46,15

42,85

49,45

44,35

41,04

32,08

72,20

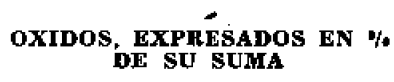
DE SU SUMA

StO $\quad \mathrm{Al}_{2} \mathrm{O}_{8} \quad \mathrm{CaO}$

31,57

31,01

28,76

32,29

28,43

31,71

28,98

29,72

29,61

23,33

33,70

26,48
34,60

24,90

24,88

43,68

35,45

24,51

44,51

38,10

28,87

41,21

33,69

33,98

34,79

47,42

42,63

33,07

33,21

33,33

39,03

42,14

48,29

25,42

14,58
14,10
12,16
13,41
11,81
12,91
9,78
9,99
8,86
$\mathbf{6 , 2 6}$
8,53
6,16
7,10
4,64
4,63
7,86
$\mathbf{6 , 2 7}$
$\mathbf{3 , 7 6}$
6,68
5,39
$\mathbf{3 , 8 8}$
$\mathbf{5 , 1 2}$
4,09
4,02
4,00
$\mathbf{5 , 3 1}$
4,76
$\mathbf{3 , 6 7}$
$\mathbf{3 , 6 5}$
$\mathbf{3 , 6 4}$
$\mathbf{3 , 9 1}$
$\mathbf{3 , 7 0}$
$\mathbf{4}, 10$
1,38

\section{4,58}

14,10

13,41

11,81

12,91
9,78

9,99

6,26

8,53

7,10

4,64

4,63
7,86

6.27

3,76

6,68

$\mathbf{3 , 8 8}$

5,12

4,09

4,02

4,76

3,67

3,65

3,64

3,70

1,38

53,85
54,89
59,08
54,30
59,76
55,38
61,24
60,29
60,53
70,41
57,77
67,36
58,30
70,46
70,49
48,46
58,28
71,73
48,81
56,51
67,25
53,67
62,22
$62,-$
61,21
47,27
52,61
63,26
63,14
63,03
57,06
54,16
47,61
73,20

18,55 
T A B L A N U M . 7

Segunda serie de ensayos

Resultado del ensayo Anstett

MUESTRA

21
22
23
23
24
25
26
27
28
29
30
31
32
33
34
35
36
37
38
39
40
41
42
43
44
45
46
47
48
49
50
51
52
53
54

AUMENTO PORCENTUAL DEL DIAMETRO A LOS DIVERSOS

\begin{tabular}{|c|c|c|c|c|c|}
\hline 7 dias & 28 dias & 2 meses & 3 meses & 6 meses & I มี̂์ \\
\hline 4,05 & 6,38 & 10,12 & 17,25 & 17,80 & 17,80 \\
\hline 4,30 & 7,90 & 8,10 & 16,15 & 17,27 & 17,50 \\
\hline $3,-$ & 3,90 & $6,-$ & 9,90 & 16,53 & 16,53 \\
\hline 2,85 & 3,72 & 5,87 & 8,60 & 10,10 & $11, \ldots$ \\
\hline 6,12 & 13,30 & $22,-$ & $22,-$ & $22,-$ & 22 \\
\hline $2,-$ & 3,94 & $7,-$ & 10,35 & 13,83 & 14,20 \\
\hline 3,30 & 7,86 & 9,50 & 9,80 & 13, & $14,-$ \\
\hline 0,75 & 3,23 & 5,90 & 8,93 & 13,40 & 15,60 \\
\hline 0,80 & 2,10 & 5,75 & 8,94 & 14,06 & 14,20 \\
\hline $2, \ldots$ & 5,60 & 10 & $11,-$ & 15,60 & 18, \\
\hline 0,83 & 1,72 & 1,93 & $2,-$ & $2,-$ & $2, \ldots$ \\
\hline 0,10 & 0,33 & 1,40 & 3,10 & 5,60 & 10,80 \\
\hline 0,14 & 0,22 & 0,25 & 0,30 & 0,30 & 0,30 \\
\hline $0,-$ & 0,17 & 0,20 & 0,37 & 1,80 & 6,58 \\
\hline $0,-$ & 0,27 & 0,56 & 0,74 & 2,10 & 6, \\
\hline $0, \ldots$ & $0,-$ & $0,-$ & 0 & 0,05 & 0,20 \\
\hline $0,-$ & 0,03 & 0,03 & 0,03 & 0,03 & 0,03 \\
\hline $0,-$ & 0,12 & 0,30 & 0.43 & 2,30 & 5,43 \\
\hline $0,-$ & $0,-$ & $0,-$ & 0,05 & 0,05 & 0,05 \\
\hline 0,10 & 0,12 & 0,13 & 0,16 & 0,18 & 0,30 \\
\hline $0,-$ & $0,-$ & $0,-$ & $0,-$ & $0,-$ & $0,-$ \\
\hline 0,05 & 0,05 & 0,05 & 0,06 & 0,20 & 0,20 \\
\hline $0,-$ & 0,10 & 0,15 & 0,16 & 0,20 & 0,40 \\
\hline $0,-$ & 0,04 & 0,05 & 0,05 & 0,05 & 0,06 \\
\hline $0,-$ & $0,-$ & 0,06 & 0,08 & 0,09 & 0,08 \\
\hline $0,-$ & 0, & $0,-$ & $0,-$ & $0,-$ & 0,06 \\
\hline $0,-$ & 0 ,- & $0,-$ & 0,47 & 0,80 & 0,80 \\
\hline $0,-$ & 0 & $0, \ldots$ & 0 & $0, \ldots$ & 0,13 \\
\hline $0,-$ & 0,10 & 0,10 & 0,10 & 0,10 & 0,10 \\
\hline $0,-$ & 0,10 & 0,10 & 0,10 & 0,10 & 0,10 \\
\hline $0,-$ & $0,-$ & $0,-$ & $0,-$ & $0,-$ & 0,08 \\
\hline $0,-$ & $0,-$ & $0,-$ & $0,-$ & $0,-$ & 0, \\
\hline $0,-$ & 0 - & $0,-$ & $0,-$ & 0,06 & 0,0 \\
\hline $0,-$ & 0,11 & 0,12 & 0,13 & 0,13 & 0,13 \\
\hline
\end{tabular}

Los resultados obtenidos se encuentran en las tablas 6 y 7. De su examen puede advertirse que también para esta serie de prueba el límite máximo de expansión a los tres meses del ensayo Anstett se alcanza con una relación $\mathrm{SiO}_{2} / \mathrm{Al}_{2} \mathrm{O}_{3}$ de alrededor de 5 . Para valores inferiores los cementos maniflestan una expansín mayor, que aumenta al disminuir dicha relación. La resistencia química al ensayo Anstett resulta "invece" absoluta, como para la serie precedente, con valores $\mathrm{SiO}_{z} / \mathrm{Al}_{2} \mathrm{O}_{3}$ superiores a 6 .

Sólo las muestras 34, 35 y 38, formadas de cemento Portland de módulo silícico elevado, tienen una marcha anormal, manifestando una cierta expansión para conservación más prolongada.

Para apreciar mejor los resultados conjuntos de la experimentaclón, los autores han representado sobre un diagrama ternario, $\mathrm{SiO}_{3}-\mathrm{Al}_{2} \mathrm{O}_{2}-\mathrm{CaO}$, los puntos representativos de la cantidad de óxidos solubles, según la técnica adaptada, presente en los distintos cementos estudiados.

Del examen de la flgura 1 se observa que el campo de composiciones posibles para los tipos de cemento considerados se subdivide en dos zonas, correspondientes a los cementos resistentes y a los no resistentes, de una manera bastante precisa.

Del gráfico resulta, pues, evidente la infuencla de la cal sobre la resistencla química de los cementos estudiados, en el sentido de que, alumentando su cantidad, es necesario, en líneas generales, relaciones $\mathrm{SiO}_{2} / \mathrm{Al}_{2} \mathrm{O}_{2}$ mayores para obtener cementos resistentes.

Una vez conftrmada la influencia favorable ejercida por la sflice reactiva en los cementos puzolánicos sobre su resistencia a las aguas sulfatadas, los autores han comenzado una serie de ensayos, todavia en curso, para aclarar el mecanismo de tal acción. 


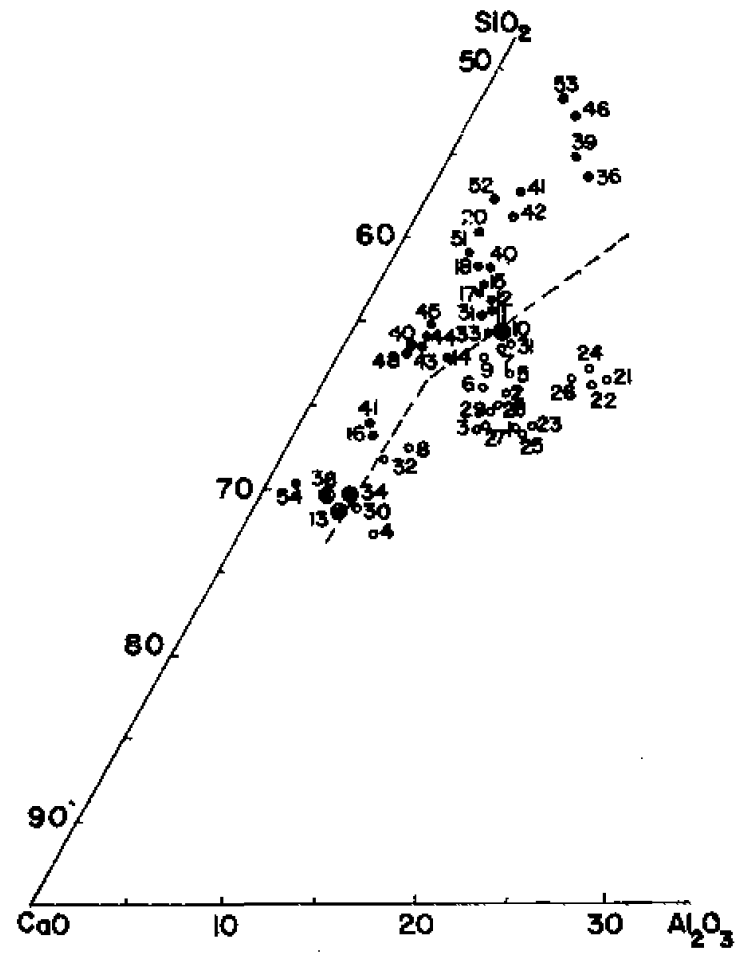

- CEMENTO NO RESISTENTE

- CEMENTOS RESISTENTES A 3 MESES

- cementos REsistentes a 12 MEses

Fig. I

Fig. 2

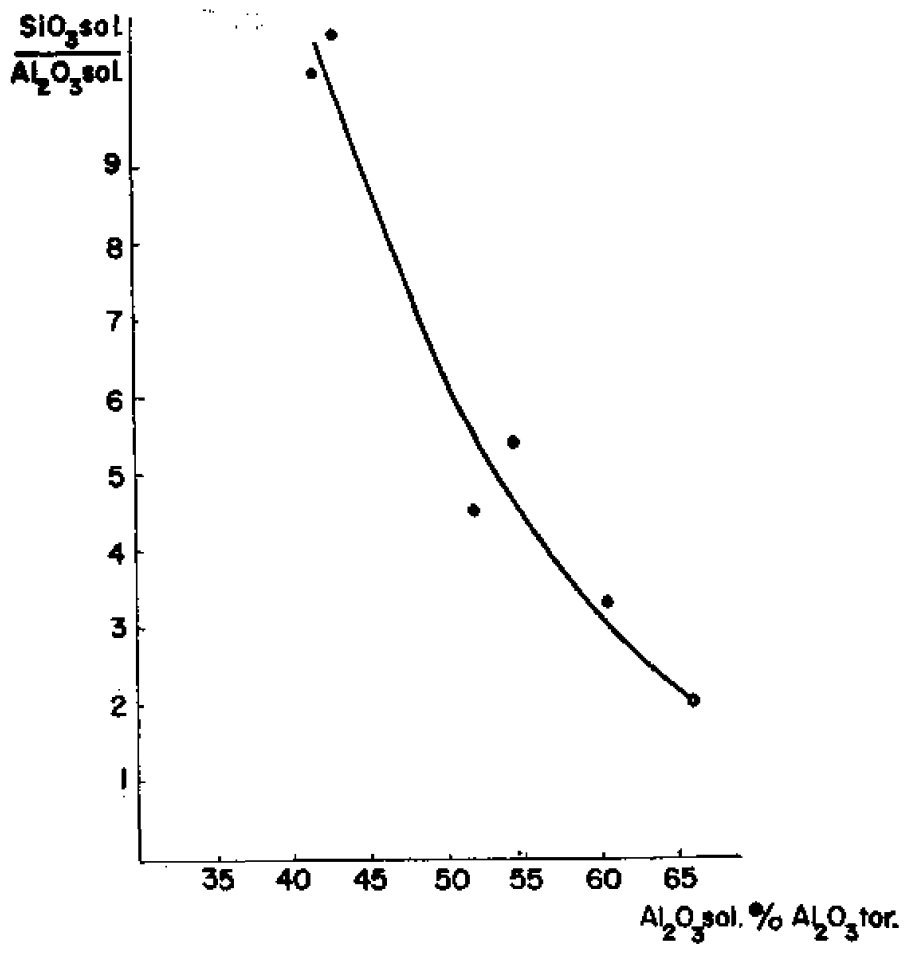

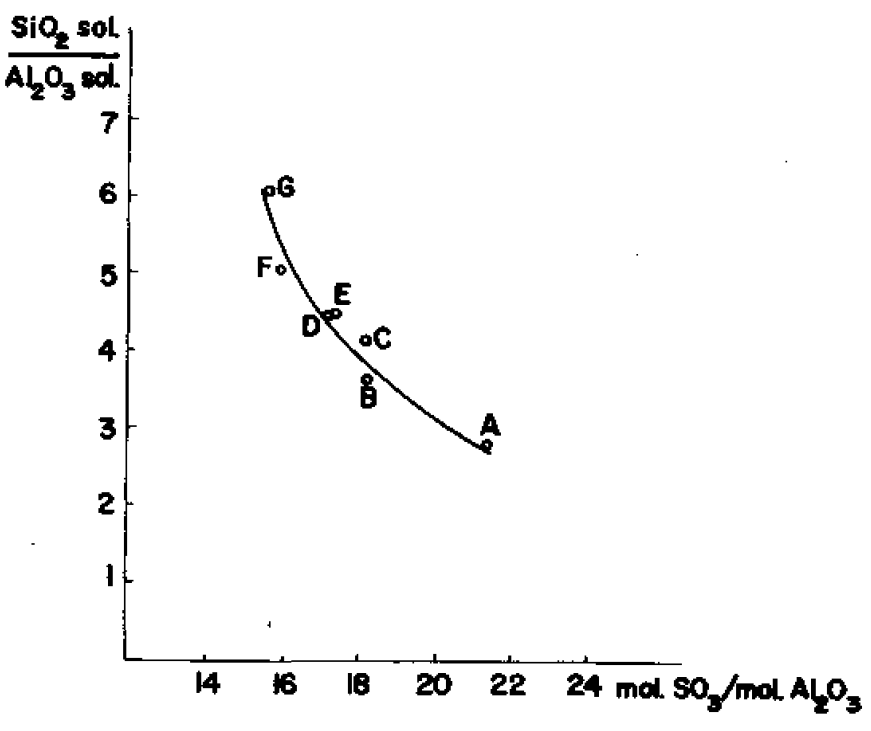

Fiz. 3

Los primeros resultados de esta investigación muestran una conflimación de cuanto se ha señalado (6); la silice reactiva de un material puzolánico presenta, frente al hidróxido cálcico, una velocidad de reacción muy superior a la de la alúmina, con el resultado de impedir, o al menos retrasar sensiblemente, la solubllización de ésta en contacto con la cal.

En la figura 2 muestra la variación de los valores de la solubilización que presenta la alúmina, en función đe la relación $\mathrm{SiO}_{2} / \mathrm{Al}_{2} \mathrm{O}_{3}$, para una mezcla de las dos puzolanas A y $B$, mantenidas en contacto con cal durante sesenta días, a temperatura amblente.

Puede observarse cómo el porcentaje de alúmina soluble, referida a la total, đisminuye progresivamente al aumentar la cantidad de sílice presente y se pasa de un valor de alrededor de $65 \%$ al de $45 \%$.

Por otra parte, una prueba de la menor susceptibilidad al ataque de los sulfatos de los aluminatos presentes en los cementos puzolánicos con elevado contenido de sílice activa, resulta de los datos expuestos en la tabla 8 y en la figura 3 , relativos a medidas de sulfatación sobre pastas de cementos puzolánicos de relaeión $\mathrm{SiO}_{2} / \mathrm{Al}_{2} \mathrm{O}_{2}$, vartable después de veintiocho días de conservación en las condiciones del ensayo Anstett. 
En tanto que para una relación $\mathrm{SiO}_{2} / \mathrm{Al}_{2} \mathrm{O}_{3}$ solubles de 2,7, la cantidad de $\mathrm{SO}_{2}$ fljada resulta de casi $12 \%$, correspondiente a una relación molar $\mathrm{SO}_{3} / \mathrm{Al}_{2} \mathrm{O}_{2}$ de 2,14 , para la muestra caracterizada por una relación $\mathrm{SiO}_{2} / \mathrm{Al}_{2} \mathrm{O}_{4}$ de cerca de 6 , desciende a 1,56 , poniendo claramente en evidencia la acción protectora que ejercen los geles de silicato cálcico hidratado presentes.

\section{T A B L A N U M , 8}

Grado de stifatación despuésde 28 días de conservación en Ias condiciones del ensayo Anstett, de pastas de cementos puzolanicos de relaciones $\mathrm{SiO}_{2} / \mathrm{Al}_{2} \mathrm{O}_{\text {a }}$ variables

(Valores referidos a muestras calcinadas)

\begin{tabular}{|c|c|c|c|c|c|}
\hline MUESTRAS & $\underset{\substack{S 10_{2} \\
\text { sotublo } \\
(\%)}}{c}$ & $\begin{array}{c}\operatorname{ACl}_{\mathbf{2}} \mathbf{O}_{s} \\
\text { soluhle } \\
\{\%\}\end{array}$ & $\begin{array}{c}\text { Reelación } \\
\mathbf{S t O}_{2} / \mathbf{A l}_{2} \mathbf{O}_{s}\end{array}$ & $\underset{\text { fijgado }}{\mathrm{SO}_{\mathrm{s}}}$ & $\begin{array}{c}\text { Relactón } \\
\text { molar } \\
\text { SOb/Aln }_{3}\end{array}$ \\
\hline $\begin{array}{l}\text { A } \\
\text { B } \\
\text { C } \\
\text { D } \\
\text { E } \\
\mathbf{F} \\
\text { G }\end{array}$ & $\begin{array}{r}19,76 \\
21,69 \\
24,54 \\
26,48 \\
23,68 \\
28,86 \\
31,83\end{array}$ & $\begin{array}{l}7,33 \\
6,05 \\
6,01 \\
5,99 \\
5,35 \\
5,75 \\
5,26\end{array}$ & $\begin{array}{l}2,70 \\
3,59 \\
4,07 \\
4,42 \\
4,43 \\
5,01 \\
6,05\end{array}$ & $\begin{array}{r}11,97 \\
8,61 \\
9,02 \\
8,49 \\
7,17 \\
7,51 \\
6,74\end{array}$ & $\begin{array}{l}2,14 \\
1,83 \\
1,83 \\
1,72 \\
1,73 \\
1,60 \\
1,56\end{array}$ \\
\hline
\end{tabular}

Como conclusión de sus trabajos, los autores señalan la posibilidad de poder prever el comportamiento de un aglomerante, frente a la acción agresiva de las aguas sulfatadas, mediante la simple determinación de la cantídad de $\mathrm{SHO}_{*}, \mathrm{Al}_{3} \mathrm{O}_{2}$ y $\mathrm{CaO}$ solubles que se encuentran en la pasta hidratada conservada en las condiciones que fija la norma para el control de los cementos puzolánicos.

Tal procedimiento, por su simplicidad y poco tiempo que requiere, puede tener un notable interés práctico, aunque sólo indicativo en cuanto, por las consideraciones expuestas, la aportaclón de resistencia se deba a la impermeabilidad del exterior de la masa.

Éxiste, además, la posibilidad, a fines de producción, de graduar, regulando oportunamente la relación de composiciones antes mencionada, la resistencia quimica de los cementos puzolánicos, hasta aicanzar una resigtencia quimica absoluta comparable a la de tipos de cementos muy especlales, tales como el sobresulfatado.

Una resistencia especifica elevada, superior a la generalmente considerada de uso ordinario, puede ser, en casos de notable agresividad, una condíción necesaria para la buena conservación de la obra con el tiempo.

Los primeros resultados de los ensayos encaminados a estudiar la causa del mejor comportamiento de los cementos que contienen un elevado porcentaje de sílice reactiva, muestran que la acción favorable de la sílice se manifiesta, o bien limitando la cantidad de alúmina presente que puede reaccionar con el hidróxido cálcico, o bien disminuyendo la susceptibilidad a los sulfatos de Los aluminatos que puedan formarse.

\section{bibliografía}

R. TuRrziani y A. Rto: "Ind. Ital. Cemento", 27, 145, (1957).

F. M. LEA: "Prolusione al IV Congresso Intermazionale sulla Chimica del Cemento". Washington, 1960.

N. Fratini: "Annali Chimica", 39, 41 (1949).

F. ARREDI: "L'Acqua", núm, 7-8 (1954).

B. Tavascr y A. Rí: "La Chimica e l'Industria", 37, 96 (1955).

R. TuRriziani y A. Rio: "Cementi pozzolanici ad alta resistenza chimica". Atti del IV Congresso Internaziom nale sulla Chimica del Cemento. Wáshington, 1960 (in corso di pubblicazione).

Consiglo Nacionale Richerem. Norme per l'accettazione dei Ieganti idraulici (1961).

L. Blondrau: "Rev. Mat, Constr.", núm. \$14, pág. $26.5(1935)_{+}$ 